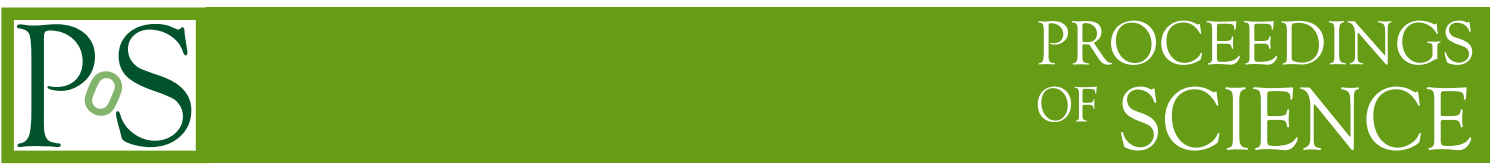

\title{
Performance of the ATLAS Trigger with Proton Collisions at the LHC
}

\author{
John Baines* \\ Rutherford Appleton Laboratory Chilton, Didcot, Oxfordshire. OX11 OQX, United Kingdom \\ E-mail: john.baines@stfC.ac.uk
}

\section{On Behalf of the Atlas Collaboration}

The ATLAS trigger has been used very successfully to collect collision data during 2009 and 2010 LHC running at centre-of-mass energies of $900 \mathrm{GeV}, 2.36 \mathrm{TeV}$, and $7 \mathrm{TeV}$. The trigger system reduces the event rate, from the design bunch-crossing rate of $40 \mathrm{MHz}$, to an average recording rate of $200 \mathrm{~Hz}$. The ATLAS trigger is composed of three levels. The first (L1) uses custom electronics to reject most background collisions, in less than $2.5 \mu \mathrm{s}$, using information from the calorimeter and muon detectors. The upper two trigger levels, known collectively as the High Level Trigger (HLT), are software-based triggers. As well as triggers using global event features, such as missing transverse energy, there are selections based on identifying candidate muons, electrons, photons, tau leptons or jets. We give an overview of the performance of these trigger selections based on extensive online running during LHC collisions and describe the progress towards fully commissioning these triggers. Distributions of key selection variables based on calorimeter and tracking information are shown calculated at the different trigger levels and are compared with offline reconstruction. Comparisons between data and simulations are shown for some important selection variables, already illustrating a very good level of understanding of the detector and trigger performance. We describe how the trigger has evolved with increasing LHC luminosity and give a brief overview of plans for forthcoming LHC running.

35th International Conference of High Energy Physics - ICHEP2010,

July 22-28, 2010

Paris France

${ }^{*}$ Speaker. 


\section{Overview}

When operating at the design luminosity of $10^{34} \mathrm{~cm}^{-2} \mathrm{~s}^{-1}$ the LHC will have a $40 \mathrm{MHz}$ bunchcrossing rate, with an average of 25 interactions per bunch crossing. The purpose of the ATLAS [1] trigger is to reduce the $1 \mathrm{GHz}$ interaction rate to about $200 \mathrm{~Hz}$ for recording and offline processing. This maximum average output rate (corresponding to $\sim 300 \mathrm{MB} / \mathrm{s}$, determined by available offline resources) can be significantly exceeded for shorter periods of time.

Detector information is stored in front-end pipelines pending a decision from the L1 trigger. In order to achieve a latency of less than $2.5 \mu \mathrm{m}, \mathrm{L} 1$ is implemented in fast custom electronics. The L1 trigger is designed to reduce the rate to a maximum of $75 \mathrm{kHz}$. The HLT consists of two levels, L2 and Event Filter (EF), implemented on farms of commodity processors connected by fast dedicated networks. The final system will consist of about $500 \mathrm{~L} 2$ nodes and $1800 \mathrm{EF}$ nodes, about half of which were installed in 2010. On a L1 accept, detector data are transferred to the Readout Buffers (RoB) which store events pending the L2 decision. The L2 selection is based on fast custom algorithms processing partial event data within the Regions of Interest (RoI) identified by L1. The RoI correspond to $2-4 \%$ of the full event data. The L2 trigger reduces the rate to $\sim 3 \mathrm{kHz}$ with an average processing time of $\sim 40 \mathrm{~ms}$. The Event Builder assembles event fragments from the RoBs for L2-accepted events, providing full event information to the Event Filter and, on a EF accept, for output to storage.

The trigger selection is configured via a trigger menu which defines trigger chains. A trigger chain specifies a sequence of reconstruction and selection algorithms which start from a L1 trigger and lead to a specific trigger signature [2]. If the requirements for one or more trigger signature are satisfied, event recording is triggered. Prescale factors can be applied to each L1 trigger and each HLT chain. A series of L1 and HLT prescale sets, covering a range of luminosities, are defined to accompany each menu. There are between 200 and 500 chains defined in the current trigger menus.

\section{Trigger Performance}

The ATLAS trigger was enabled incrementally during 2010, starting with a L1-based selection, and enabling HLT rejection as needed to match increasing LHC luminosity. At the start of 2010 running, luminosity was such that it was possible to record all collisions triggered by the L1 Minimum Bias (MBTS) trigger [3]. The MBTS trigger is based on scintillators installed in each end-cap covering the pseudo-rapidity region of $2.09<|\eta|<3.84$. The MBTS_1 trigger requires at least one counter above threshold in coincidence with a filled LHC bunch, this trigger is $99.7 \%$ efficient for collisions with at least one track with transverse momentum $\mathrm{p}_{T}>500 \mathrm{MeV}$. As luminosity increased above about $2 \times 10^{27} \mathrm{~cm}^{-2} \mathrm{~s}^{-1}$, the MBTS trigger was prescaled and other L1 triggers became the principal triggers for physics. Fig. 1 shows L1 rates as a function of instantaneous luminosity (measured in 2 minute intervals) for various triggers: MBTS_1, MBTS_4_4 (4 counts in each end-cap), electron/photon (EM), jet (J) and muon (MU) L1 triggers. The number gives the nominal threshold $(\mathrm{GeV})$. During this early period the HLT was operated in monitoring mode, with the algorithms running online but not rejecting events. This enabled validation of the HLT, such that rejection could be progressively enabled as required. The HLT was fully active from August 2010 when the luminosity reached $2 \times 10^{30} \mathrm{~cm}^{-2} \mathrm{~s}^{-1}$. The luminosity subsequently rose by 


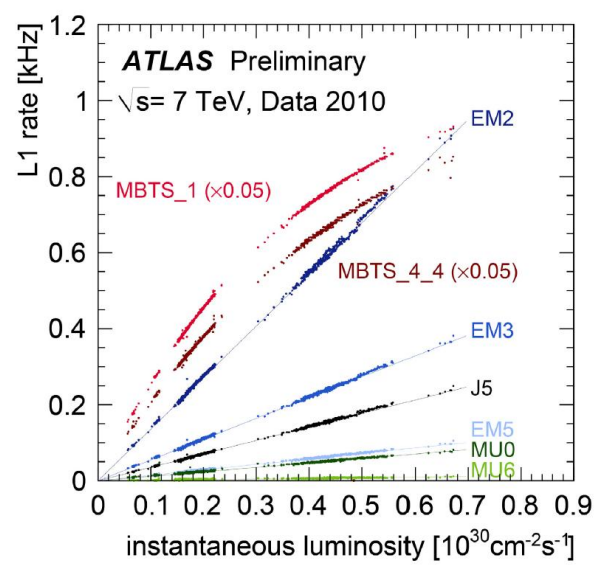

Figure 1: Rates for various L1 triggers (MBTS rates scaled by 0.05$)$. The MBTS_1 rate saturates approaching the frequency of the colliding bunches.

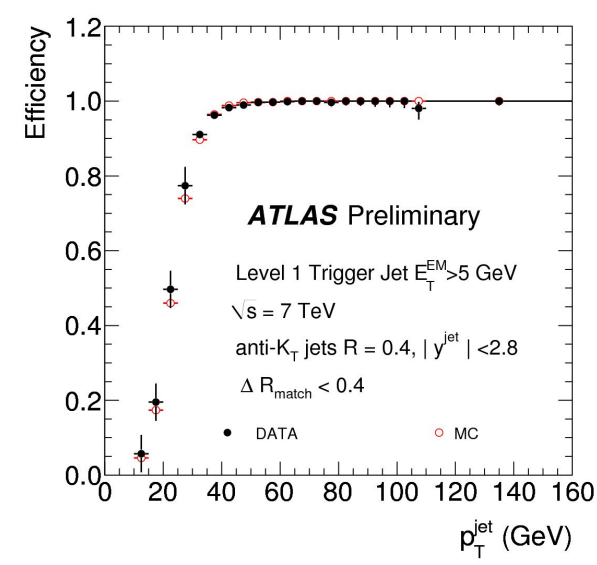

Figure 2: L1 jet trigger efficiency versus calibrated offline jet $\mathrm{p}_{T}$ shown for data and $\mathrm{MC}$ simulation.

two orders of magnitude during the remainder of the 2010 data taking period, during which time the trigger (L1 and HLT) was instrumental in selecting the data for physics.

The L1 jet trigger sums transverse energy $\left(\mathrm{E}_{T}\right)$ in the Electro-Magnetic $(\mathrm{EM})$ and hadronic calorimeters in a relatively large cone $(0.8 \times 0.8$ in $\eta \times \phi$, where $\phi$ is the angle measured in a plane perpendicular to the beam-pipe). The L1 jet trigger efficiency is shown in Fig. 2, for the lowest threshold, as a function of the calibrated offline jet $\mathrm{p}_{T}$ reconstructed using the anti-kt algorithm [4]. The lowest jet threshold is nominally $5 \mathrm{GeV}$ (the cut applied to the uncalibrated EM-scale L1 jet), but in practice full efficiency is only reached at about $40 \mathrm{GeV}$. There is good agreement between data and Monte Carlo simulation.

The L1 electron/photon trigger sums transverse energy in the EM calorimeter in a relatively small $(\eta \times \phi=0.2 \times 0.2)$ cone. The efficiency is shown as a function of offline cluster $\mathrm{E}_{T}$ in Fig. 3 for data and MC. This shows full efficiency above the threshold and good agreements with simulation. The calorimeter clustering is repeated at the HLT using full granularity information, and including information from the EM pre-sampler. Selections are applied based on various cluster shape parameters, such as $\mathrm{R}_{\eta}$ which is the ratio of transverse energy in a $3 \times 7$ window to that in a $7 \times 7$ window (dimensions in $\eta \times \phi$ using calorimeter cell units of $\Delta \eta \times \Delta \phi=0.2 \times 0.2$ ). Fig. 4 shows the distribution of $\mathrm{R}_{\eta}$ calculated at the EF for offline electron candidates. The distribution is peaked towards $\mathrm{R}_{\eta}=1$ for electrons. The shape of the distribution is well modeled by simulation. After the calorimetry-based selection, tracks are reconstructed in the ID and cluster-track matching cuts are applied. In Fig. 5 the efficiency for reconstructing tracks at L2 and the EF is shown for offline electron candidates passing the L1 trigger, demonstrating $100 \%$ efficiency for electrons with $\mathrm{p}_{T}>5 \mathrm{GeV}$.

The L1 tau trigger [5] uses a cone of $\eta \times \phi=0.2 \times 0.2$ to sum energy in the EM and Hadronic calorimeters. Isolation requirements can be imposed at L1 for both the electron/photon and tau triggers, but were not used in 2010 running. In the HLT a tau candidate is identified as a well collimated calorimeter cluster with a small number of associated Inner Detector tracks. The number of associated L2 tracks is shown in Fig. 6, demonstrating good agreement between data 


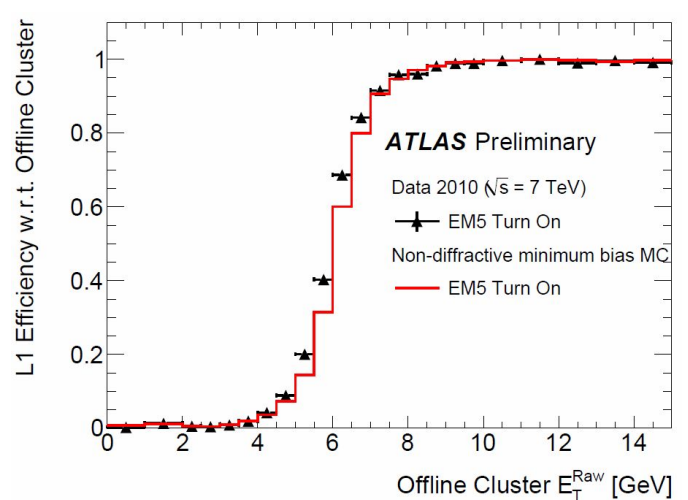

Figure 3: Efficiency versus offline cluster $\mathrm{E}_{T}$ (EM scale) for the $5 \mathrm{GeV}$ L1 EM threshold.

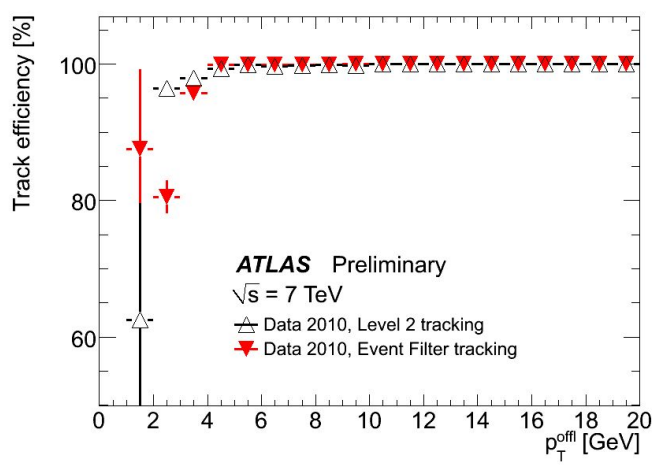

Figure 5: L2 and EF tracking efficiency for offline reconstructed electrons.

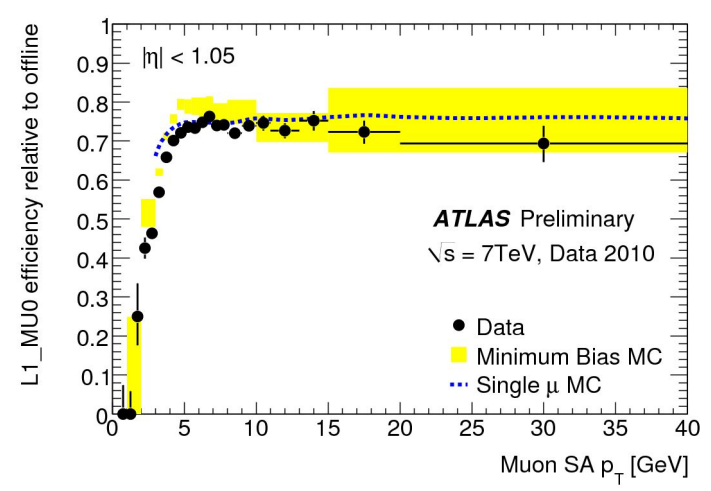

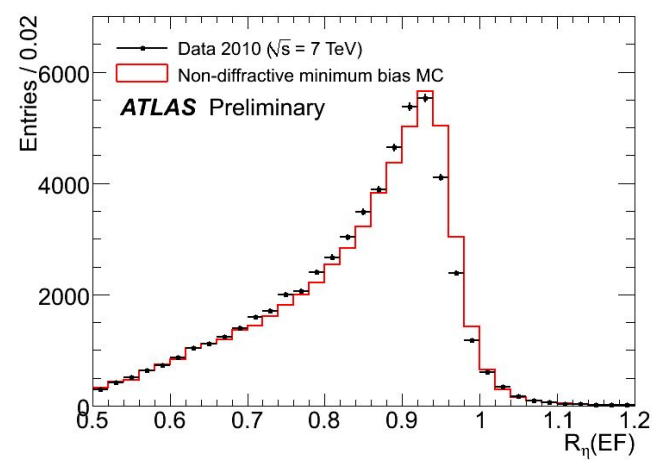

Figure 4: $\mathrm{EF}_{\eta}$ distribution for $\mathrm{MC}$ and data.

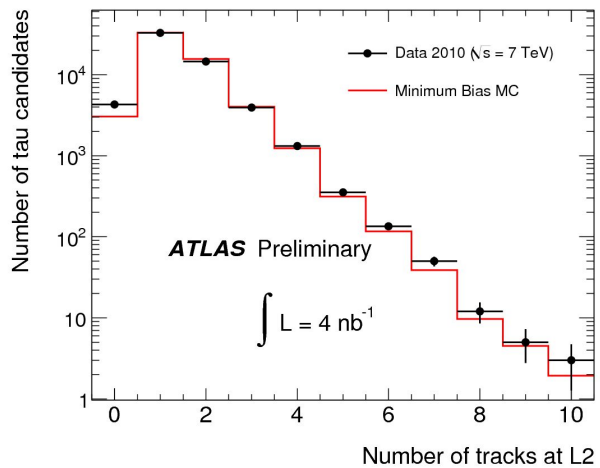

Figure 6: Distribution of the number of L2 tracks in tau RoI for data and MC.

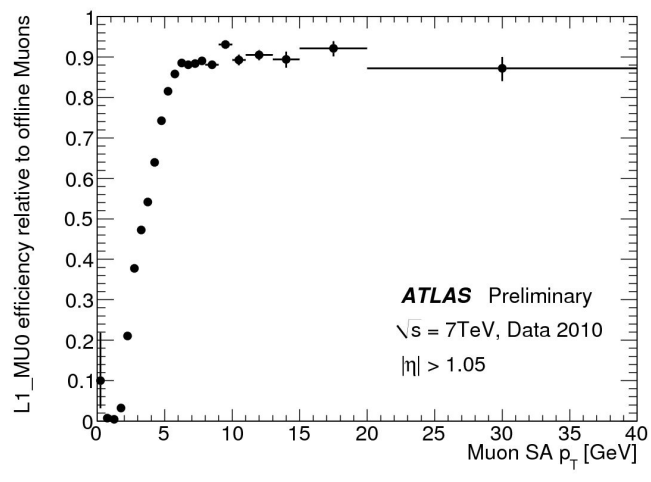

Figure 7: Efficiency versus offline muon $\mathrm{p}_{T}$ for the lowest threshold RPC (left) and TGC (right) triggers. 
and simulation.

The L1 muon trigger is based on dedicated detectors; Resistive Plate Chambers (RPC) in the barrel $(|\eta|<1.05$ ) and Thin Gap Chambers (TGC) in the end-caps. The RPC efficiency is shown as a function of offline muon $\mathrm{p}_{T}$ for the lowest trigger threshold in Fig. 7 (left). This includes a loss of $\sim 20 \%$ due to support structures etc. The corresponding TGC efficiency is shown in Fig. 7 (right). At the HLT, the L1 trigger is confirmed using information from the precision muon detectors to form muon track segments. The efficiency for reconstructing tracks segments is more than $98 \%$ with repect to $\mathrm{L} 1$ for muons with $\mathrm{p}_{T}>4 \mathrm{GeV}$. Muon segments are then combined with Inner Detector tracks to form combined muon candidates.

In addition to the RoI-based triggers, there are L1 Missing $\mathrm{E}_{T}$ (MET) and Total $\mathrm{E}_{T}$ (TE) triggers based, respectively, on vector and scalar sums of calorimeter cell energy over the entire calorimeter. At L2 they are corrected using muon information and at the EF they are recalculated using information from both the calorimeter and muon detector. Up to a luminosity of $\sim 10^{30} \mathrm{~cm}^{-2} \mathrm{~s}^{-1}$, it was possible to run with an un-prescaled $10 \mathrm{GeV}$ MET threshold. This was raised, in steps, to $30 \mathrm{GeV}$ as luminosity rose to $2 \times 10^{32} \mathrm{~cm}^{-2} \mathrm{~s}^{-1}$. In addition to single MET items, the menu contains combined triggers such as tau plus MET used, for example, as a trigger for $\mathrm{W} \rightarrow \tau \nu$.

\section{Summary}

The ATLAS trigger has been successfully commissioned and has been instrumental in delivering the data for ATLAS physics analysis. There is generally excellent agreement between quantities calculated online, using fast trigger algorithms, and those reconstructed offline. There is also good agreement between data and MC simulation for these quantities and for measured trigger efficiencies. The trigger has been demonstrated to function very well, meeting operational requirements and evolving to meet the demands of rapidly increasing LHC luminosity. The trigger selection will continue to evolve to meet future demands via progressive increase of pre-scales, tightening of selection cuts, application of isolation requirements as appropriate and increased use of higher multiplicity and multi-object triggers. As a result of the very good performance of the trigger in this years running and offline studies confirming the scaling to higher luminosities, we are confident that the trigger will continue to meet the challenges of running in 2011 and beyond.

\section{Acknowledgements}

We wish to thank CERN for the efficient commissioning and operation of the LHC during this initial high-energy data-taking period as well as the support staff from our institutions without whom ATLAS could not be operated efficiently.

We acknowledge the support of ANPCyT, Argentina; YerPhI, Armenia; ARC, Australia; BMWF, Austria; ANAS, Azerbaijan; SSTC, Belarus; CNPq and FAPESP, Brazil; NSERC, NRC and CFI, Canada; CERN; CONICYT, Chile; CAS, MOST and NSFC, China; COLCIENCIAS, Colombia; MEYS (MSMT), MPO and CCRC, Czech Republic; DNRF, DNSRC and Lundbeck Foundation, Denmark; ARTEMIS, European Union; IN2P3-CNRS, CEA-DSM/IRFU, France; GNAS, Georgia; BMBF, DFG, HGF, MPG and AvH Foundation, Germany; GSRT, Greece; ISF, 
MINERVA, GIF, DIP and Benoziyo Center, Israel; INFN, Italy; MEXT and JSPS, Japan; CNRST, Morocco; FOM and NWO, Netherlands; RCN, Norway; MNiSW, Poland; GRICES and FCT, Portugal; MERYS (MECTS), Romania; MES of Russia and ROSATOM, Russian Federation; JINR; MSTD, Serbia; MSSR, Slovakia; ARRS and MVZT, Slovenia; DST/NRF, South Africa; MICINN, Spain; SRC and Wallenberg Foundation, Sweden; SER, SNSF and Cantons of Bern and Geneva, Switzerland; NSC, Taiwan; TAEK, Turkey; STFC, the Royal Society and Leverhulme Trust, United Kingdom; DOE and NSF, United States of America.

\section{References}

[1] The ATLAS Collaboration, The ATLAS Experiment at the CERN Large Hadron Collider, JINST, 3 (2008) S08003 (2008) [doi: 10.1088/1748-0221/3/08/S08003]

[2] S. George for the ATLAS Collaboration, The ATLAS High Level Trigger Configuration and Steering Software: Experience with $7 \mathrm{TeV}$ Collisions, In these proceedings.

[3] R. Kwee for the ATLAS Collaboration, Minimum Bias Trigger in ATLAS In these proceedings.

[4] M.Cacciari et al., The anti-kt jet clustering algorithm, JHEP, 804 (2008) p63

[5] M. Shamim for the ATLAS Collaboration, Performance of the ATLAS tau trigger with $7 \mathrm{TeV}$ collision data at the LHC, In these proceedings. 